\title{
Comprensión didáctica del currículo como espacio público
}

\section{Didactic understanding of the curriculum as a public space}

\author{
Compreensão didática do currículo como espaço public
}

\author{
Betsi Fernández*a \\ ${ }^{a}$ Doctora en Ciencias de la Educación, Universidad Pedagógica experimental \\ libertador; Venezuela. (D)
}

Forma de citar: Contreras, A. (2018). Precisiones conceptuales y procedimentales acerca de la preposición de aproximaciones teóricas en las tesis doctorales. Perspectivas, 3(1). 59-70

Recibido: mayo 07 de 2017

Aceptado: agosto 24 de 2017

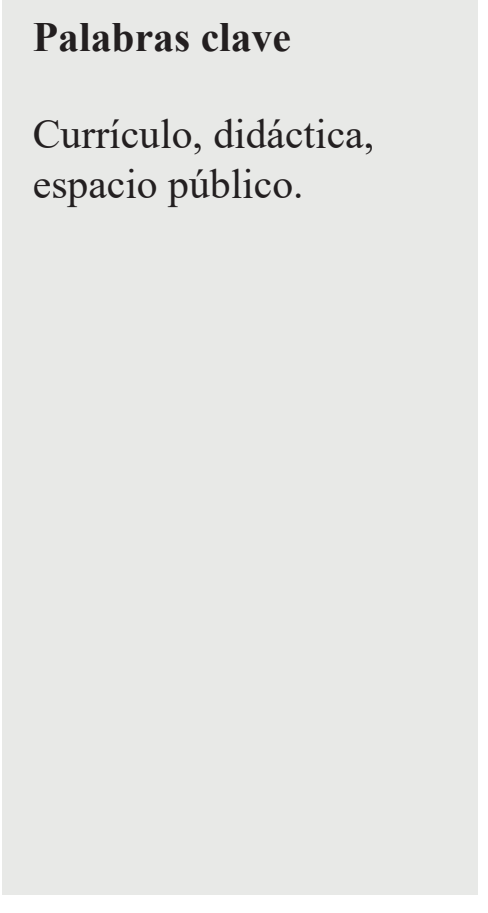

\section{Palabras clave}

Currículo, didáctica, espacio público.

* Autor para correspondencia doloresp@bu.edu
Resumen: El presente estudio, referido a la dinámica curricular del siglo XXI en la Universidad Pedagógica Experimental Libertador (UPEL), en Venezuela, tiene como propósito generar criterios para la comprensión didáctica y contextual del currículo como espacio público en la institución como contribución con la gestión integral del proceso formativo de los ciudadanos, profesionales e investigadores y la distribución del poder en la construcción del diseño curricular de la carrera docente y áreas afines con sentido social, cultural, histórico y ético. Los propósitos específicos han sido: (a) comprender el conocimiento curricular como producción intelectual condicionada por el contexto social y el momento histórico que vive la UPEL, (b) describir el proceso de legitimación del discurso curricular al concebir el currículo como espacio público, desde el debate de las ideas y los valores contextuales presentes en la UPEL. La investigación está enmarcada en el paradigma naturalista, bajo un enfoque cualitativo, en una modalidad de investigación de campo, descriptiva y documental. Se emplean como técnicas la entrevista y el análisis de contenido y como instrumentos el guion de entrevistas y la matriz de análisis de contenido. Los resultados se analizan cualitativamente, aplicando la triangulación de fuentes para generar los criterios orientadores de la comprensión didáctica y contextual del currículo como espacio público, producto de la investigación.

https://doi.org/10.22463/25909215.1424 


\section{Keywords}

Curriculum, didactics, public space.

\section{Palavras chave}

Currículo, didática, espaço público.
Abstract: The present study, referred to the curricular dynamics of the XXI century in the Universidad Pedagógica Experimental Libertador (UPEL), in Venezuela, has the purpose of generating criteria for the didactic and contextual understanding of the curriculum as a public space in the institution as a contribution to integral management of the formative process of citizens, professionals and researchers and the distribution of power in the construction of the curricular design of the teaching career and related areas with a social, cultural, historical and ethical sense. The specific purposes have been: (a) to understand the curricular knowledge as intellectual production conditioned by the social context and the historical moment that UPEL lives, (b) to describe the process of legitimization of the curricular discourse when conceiving the curriculum as a public space, from the debate of the ideas and the contextual values present in the UPEL. The research is framed in the naturalist paradigm, under a qualitative approach, in a field, descriptive and documentary research modality. The interview and the content analysis are used as techniques and the interview script and the content analysis matrix as instruments. The results are analyzed qualitatively, applying the triangulation of sources to generate the guiding criteria for the didactic and contextual understanding of the curriculum as a public space, the product of research.

Resumo: Este estudo, com base na dinâmica curricular do século XXI na Universidade Pedagógica Experimental Libertador (UPEL) na Venezuela, visa gerar critérios para o ensino ea compreensão contextual do currículo como um espaço público na instituição como uma contribuição para a gestão integral do processo formativo dos cidadãos, profissionais e pesquisadores e a distribuição de poder na construção do desenho curricular da carreira docente e áreas afins com sentido social, cultural, histórico e ético. Os objetivos específicos foram: (a) entender o conhecimento curricular como a produção intelectual condicionada pelo contexto social e momento histórico na UPEL, (b) descrever o processo de legitimação do discurso curricular na concepção do currículo como um espaço público, a partir de o debate das ideias e os valores contextuais presentes na UPEL. A pesquisa enquadra-se no paradigma naturalista, sob abordagem qualitativa, em uma modalidade de pesquisa de campo, descritiva e documental. A entrevista e a análise de conteúdo são utilizadas como técnicas e o roteiro de entrevista e a matriz de análise de conteúdo como instrumentos. Os resultados são analisados qualitativamente, aplicando a triangulação de fontes para gerar os critérios norteadores para a compreensão didática e contextual do currículo como espaço público, produto da pesquisa. 


\section{Introducción}

La problematización contextual es la manera de recuperar lo potencial de la realidad Zemelman (2006:119).

La constante búsqueda de respuestas a las interrogantes humanas a través del tiempo ocupa la razón de ser del contexto curricular en las instituciones universitarias, corresponsables de la formación integral de los ciudadanos profesionales e investigadores que se requieren en cualquier sociedad y de manera muy particular al intentar responder a los requerimientos generados a partir de las tendencias emergentes a nivel mundial, en la educación superior (educación universitaria en el caso venezolano). Entre estas tendencias pueden mencionarse las enunciadas por Brunner (2014): a) masificación del acceso a la educación universitaria, b) diferenciación del campo organizacional, c) situación de la docencia y de la investigación, d) globalización e internacionalización, e) tecnología y educación a distancia. Estas particularidades, como es natural, deberían ser consideradas a la hora de generar construcciones curriculares pertinentes, flexibles, contextualizadas, diversas y de calidad.

Se puede afirmar que una de las prioridades de la educación universitaria está en desarrollar propuestas curriculares claras, coherentes, con procesos formativos que además de favorecer el acceso, también favorezcan la permanencia de los alumnos mediante oportunidades que aseguren el aprendizaje del estudiante y en consecuencia, el desarrollo de sus capacidades, convirtiéndolos en personas competentes, con un desempeño idóneo, capaz de identificar, describir, interpretar y comprender la realidad para poder transformarla, empleando adecuadamente las tecnologías y el lenguaje propios del área de conocimiento en la que se forme, se relacione e interactúe con grupos heterogéneos y sea responsable y autónomo en todo su desempeño.

Desdeestavisión compartidacon investigadores del ámbito educativo y fundamentalmente del universitario (p.e.: Brunner, 2014; Becerra,1974, 2004: Zemelman,2006, 2011), se presenta esta investigación como resultado de la búsqueda de alternativas que contribuyan al mejoramiento de la formación de ciudadanos, profesionales e investigadores. Bajo un paradigma naturalista, se explora una forma de hacer currículo y de viabilizar su concreción desde la comprensión didáctica del currículo como espacio público, propósito fundamental de este trabajo. Asimismo, entre los propósitos de la investigación se encuentra la descripción del proceso de legitimación del discurso curricular al concebir el currículo como espacio público, desde el debate de las ideas y los valores contextuales presentes en la UPEL en la construcción del conocimiento curricular del siglo XXI.

\section{Problematización contextual: comprensión didáctica y contextual del currículo.}

\section{Es más fácil no hacerse preguntas o creer en las respuesta de otro. Zemelman (2006:80).}

La rehabilitación social, política, cultural y educativa emprendida en el siglo XXI por la sociedad venezolana parece no ser suficiente para dar por agotada la discusión en torno a la educación universitaria, necesaria para la formación del ciudadano profesional e investigador venezolano que demanda la realidad. Por ello, se comparte la idea de que las reformas curriculares solo han logrado refrescar y mantener básicamente el mismo modelo anterior, sin mayor exigencia que la demanda presupuestaria. En este sentido, se puede afirmar que en Venezuela, aunque se tiene una nueva Constitución desde 1999 y una nueva Ley Orgánica de Educación, promulgada en el año 2000, se sigue manteniendo el mismo currículo en casi todas las instituciones universitarias. Una de las excepciones es la Universidad Pedagógica Experimental Libertador (UPEL), la cual cuenta con un nuevo currículo desde el año 2011 (Consejo 
Universitario 308 de mayo de 2011) y con nuevos Diseños Curriculares sancionados por el Consejo Universitario (años 2015 y el 2016).

Lo anterior evidencia que la dinámica curricular en las instituciones universitarias venezolanas constituye un escenario de actuación, interacción y comunicación constante en la cotidianidad de quienes tienen la responsabilidad de formar al ciudadano que se requiere para el siglo XXI. Independientemente del nivel, modalidad o campo de conocimiento en el cual se desempeñen los actores, las demandas curriculares deberían estar a la par para adaptarse a los procesos de cambio históricos, políticos, sociales, culturales, económicos y legales que desde la década de los noventa se fortalecen en América Latina y en consecuencia en Venezuela.

En materia curricular, las instituciones universitarias se mantienen en alerta académica, abordan las demandas sociales, con rigor esencialmente investigativo, abierto, flexible, autónomo, concertado, democrático y transformador, para dar cuenta del nivel de apropiación y conocimiento del discurso curricular que las identifica según sea su naturaleza, dando evidencias de su status en esta materia y quedando develada la necesidad de conocer, identificar, interpretar y comprender la cultura curricular que las identifica.

La sociedad exige a las instituciones universitarias respuestas oportunas, claras y coherentes expresadas en ofertas educativas heterogéneas, flexibles, diversas y contextualizadas. Estas exigencias recaen de manera especial sobre las instituciones encargadas de la formación docente, las cuales deben asumir el compromiso de presentar ofertas contextualizadas, derivadas de un proceso investigativo sobre las demandas potenciales de docentes que respondan a los requerimientos específicos de desarrollo, por encontrarse inmersas en un cambio fundamental de los fines de la educación, (LOE 2000) expresados en una nueva concepción del ser de la escuela, la educación y la sociedad, en cuyo seno se sitúan las categorías que definen la acción educativa. A ello se aboca fundamentalmente la Universidad Pedagógica Experimental Libertador (UPEL), al precisar las necesidades de formación de docentes, desde los pueblos originarios hasta las capitales de estados, dada su ubicación y amplia distribución en el territorio nacional.

Las exigencias de la sociedad del siglo XXI encuentren en la UPEL evidencias del imaginario de los actores involucrados en una cultura curricular que aborda lo cotidiano de cada quien y el contexto en el que dichos imaginarios permiten que haya sentido para la acción humanizadora del proceso curricular. Iniciado solo como proceso de reforma curricular en la década de los noventa 1996, ha proseguido como proceso de modernización y transformación curricular desde la primera década del nuevo milenio, específicamente a partir del año 2006, cuando se enfatiza en la modernización y en la transformación curricular desde el Vicerrectorado de Docencia.

Con este giro se espera que la actividad curricular trascienda e impacte de tal manera que se pueda desde allí dar inicio al proceso de trasformación universitaria, donde se desplace el acento de una administración centrada en los recursos a una administración centrada en los procesos, que privilegie entre otros aspectos a la docencia como una función que abarca tanto al pregrado como el postgrado.

Así, la necesidad de transformar las instituciones universitarias como proceso de legitimación del cambio emergente en el siglo XXI, llega a la Universidad Pedagógica Experimental Libertador como consecuencia de la evaluación institucional realizada durante los años 20042005 en sus ocho Institutos Pedagógicos: Instituto Pedagógico de Caracas, Instituto Pedagógico de Barquisimeto "Luis Beltrán Prieto Figueroa", Instituto Pedagógico de Maturín, Instituto Pedagógico de Maracay "Rafael Alberto Escobar Lara", Instituto Pedagógico de Miranda "José Manuel Siso Martínez", Instituto de Mejoramiento Profesional del Magisterio, Instituto Pedagógico 
Rural "El Mácaro" y el Instituto Pedagógico Rural "Gervasio Rubio".

Los resultados de la evaluación institucional realizada señalaron con claridad la importancia de valorar todos los elementos que conforman la Universidad, incluyendo el currículo de pregrado y de postgrado, el primero referido durante el desarrollo del estudio, como fundamental para la comprensión de la dinámica universitaria en la UPEL, y el segundo, como vía para la profundización y el perfeccionamiento (Fernández, 2006, 2007).

En este sentido y dado que tanto en el documento de Políticas y Estrategias para el desarrollo de la Educación Superior en Venezuela 2000-2006 del Ministerio de Educación Cultura y Deportes (2001), como en la VII Reunión Nacional de Currículo y en el Congreso Internacional sobre Calidad e Innovación en Educación Superior celebrado en Caracas, del 9 al 13 de abril de 2007, se demanda la transformación universitaria, en la UPEL, se procede a formular el proyecto de Modernización y Transformación del Currículo de Pregrado, bajo la conducción del Vicerrectorado de Docencia durante el período 2006-2009.

A partir de este momento, se inicia la revisión de propuestas innovadoras negociadas con los actores, a través del diálogo y el respeto como dimensiones éticas fundamentales presentes en los enunciados iniciales para configurar la problematización contextual de la cultura curricular, que hasta finales de la primera década del 2000, fue considerada territorio exclusivo de unos pocos, que con dominio del discurso curricular, hacían suyo el currículo. Aquello trajo como consecuencia que el discurso curricular utilizado dificultara la comunicación y la comprensión del currículo, al punto de generar en algunos casos rechazo, distancia y hasta exclusión. En otro sentido, esta postura tradicionalista privilegiana a aquellos que por costumbre o tradición se dedicaron a reproducir las ideas de autores foráneos sin ningún esfuerzo por promover su contextualización.
Indagar sobre la realidad de la construcción curricular en la UPEL, lleva a enfrentar las características de las condiciones sociales, históricas, políticas, culturales, contextuales, pedagógicas y didácticas, en las formas de relacionarse los unos con los otros a la hora de elaborar un diseño curricular, mostrando restricciones y descontento como los manifestados por los actores en los diferentes institutos cuando se llevó a cabo el rediseño curricular de 1996 y ahora, cuando se inicia el proceso de transformación curricular. Al respecto se señalan algunas de las afirmaciones expuestas por los informantes clave, uno por instituto, al consultar su opinión con relación a la participación de los docentes en la elaboración de los Diseños Curriculares de las diferentes Especialidades.

Informante clave A:

Venimos de la experiencia de las reuniones de Pozo de Rozas, llevamos una cosa y luego el producto presentado en el Documento Base del Diseño Curricular del 96 fue otra. Claro, eso pasó porque solo fueron unos cuantos privilegiados a llevar las propuestas y otros decidieron que hacer por nosotros, dígame Profe: ¿es verdad que ya los diseños nuevos están listos? La participación de la mayoría de nosotros fue casi nula, nos llegaron con un discurso curricular, en verdad Profe, y me disculpa, que solo ustedes entienden. Son docentes pero explican para ustedes mismos, sin ninguna pedagogía. Lo ideal sería que cualquier actividad vinculada al currículo fuera comprendida por todos; pero, no es así.

Informante clave B:

Yo creo que no aplicaron criterios de equidad. Al instituto con más trayectoria le dieron la ventaja de decidir que iba y que no iba en el Diseño Curricular. Eso no es justo. Todos

${ }^{1}$ Centro de Convenciones próximo a Caracas. 
tenemos derecho a opinar. ¿No cree usted profe? Claro, en los institutos con más trayectoria hay favoritismo. Como ellos son los que saben de currículo, bueno, dicen ellos... En realidad todos deberíamos saber, ya que lo aplicamos o administramos cuando damos clases, cuando planificamos, por ejemplo, tenemos que saber para qué planificamos. Sin embargo, la participación de nosotros fue muy restringida, nada de revisar todo esto que ustedes piden, que si enfoques, tendencias, perspectivas del área de conocimiento en la que uno se desempeña. Anteriormente, nuestra participación estuvo centrada en la elaboración de los programas sinópticos, muchos de los cuales no fueron culminados. $\mathrm{O}$ me equivoco?

Informante clave $\mathrm{C}$ :

Bueno... No todos los profesores participamos en la discusión porque eso se hacía en los Departamentos y los demás ni sabíamos cuándo se reunían. Los profesores de postgrado no fuimos consultados, por lo menos en mi caso, yo no fui consultado. Y solo algunos participaban; a veces, uno que otro estudiante, porque era preparador o beca trabajo. Los profesores de pregrado eran los que más o menos participaban.

\section{Informante clave D:}

En realidad, uno no entiende mucho de currículo y los que saben es mejor que no enseñen, Ja, ja, ja, ja... porque enredan más el asunto, hablan para que nadie entienda o comprenda lo que dicen. Usted me perdona, pero la gente de currículo es así. Bueno, como dicen, mejorando lo presente... Verdaderamente que lo curricular es mejor dejarlo a los expertos. A mí que me digan que hay que hacer y ya. Yo me dedico a lo mío y lo mío es mi materia. Creo que en esta oportunidad va a ser diferente. Por lo menos empezamos muy bien, con reuniones por especialidades para llegar a definir las características del profesional que se espera formar. Me parece interesante hacer el currículo desde el perfil del egresado y promover la participación de todos sin que todo venga listo desde la "Santa Sede".

\section{Informante clave E:}

Bueno... Aquí solo opinaron los llamados a la mesa, como dicen, porque la mayoría se mostró indiferente. Aquí prefieren que le digan lo que tienen que hacer y ya. Siempre preguntan ¿cuál es la tarea? ¡Y para que le expliquen a uno como no queriendo que se entienda lo explicado...! Pues es mejor que no. Ja, ja, ja, ja, ja... No es fácil comprender el trabajo en currículo, pero en realidad siento que ahora hay mayor participación, aunque se tiene la idea de que los nuevos diseños ya están listos.

Informante clave F:

Yo fui a las reuniones preparatorias de Pozo de Rozas y te puedo decir que realmente allí se decidieron cosas que no eran las que llevábamos los profesores que representábamos a cada Instituto. Creo que por lograr acuerdos entre los asistentes fue que al final se hizo un arreglo ahí y ya está. Ojalá que esta vez no sea así, aunque para muchos es mejor que ni los consulten, pues en realidad eso es mucho trabajo. Aquí hemos estado trabajando en la reforma curricular, o como usted dice, en la transformación curricular, desde hace más de cuatro años. Y es difícil llegar a puntos comunes sin discusión y análisis, lo que implica ver esta transformación como un proceso de estudio. Yo he tenido que revisar muchos materiales y hasta consultar a mis estudiantes.

Informante clave G:

Bueno... Le puedo decir que hubo diseños como el Intercultural Bilingüe que ni siquiera fue revisado. Bueno, ya usted debe haberlo visto, porque tiene tiempo en esto, y habrá visto que 
permanece igual desde la década de los ochenta. No sé cómo se ha mantenido así, sin ninguna modificación, a pesar de los cambios educativos que se han dado. Tanto en las orientaciones pedagógicas como didácticas, y aunque se estén asumiendo, en realidad no hay forma de saberlo, pues eso no está escrito en ninguna parte. Creo que desde la unidad de currículo se debe impulsar el cambio, pero de forma sencilla y clara, y no con un discurso rebuscado que realmente resulta complejo sobre todo para los que no somos docentes de profesión, los cuales hacemos un esfuerzo por comprender lo que nos están solicitando.

Informante clave $\mathrm{H}$ :

¡Qué va Profe! Aquí se nombró una comisión que fue la que se encargó de trabajar en eso, siguiendo las instrucciones de los expertos. Ja, ja, ja, ja... Me imagino que en su Instituto también fue así, y bueno, ¡como es el de Caracas! Ja, ja,ja, ja... Le dieron más chance de meter materias y eso. En realidad, espero que con las estrategias propuestas para la transformación la situación cambie y se garantice la participación, que las opiniones sean tomadas en cuenta, como se vio en el evento realizado en El Mácaro. Fue algo bien significativo, porque todos nos sentimos tomados en cuenta".

\section{Discusión y análisis de los resultados}

De lo expuesto se pueda afirmar que el desarrollo curricular en la UPEL se presenta en el siglo XXI aun bajo el dominio de los expertos tradicionales, que mantienen el control del escenario curricular sin propiciar la comprensión y apropiación por la mayoría de los actores, que, de acuerdo con los registros de investigación en reuniones y entrevistas con profesores de diferentes especialidades y en entrevistas realizadas, señalan "no saber nada de currículo y no tener conocimiento curricular más allá de lo que asumen en su actividad académica, limitada a dar clases".

Expresiones tales como "a la gente de currículo nadie le entiende", "utilizan palabras que nadie sabe qué significan", "creen que saben más que todo el mundo "se dejan escuchar en reuniones institucionales en las que participan estudiantes y profesores bajo la conducción de Jefes de Currículo que solicitan información necesaria para nutrir una serie de documentos sistemáticos que sin llegar a ser estandarizados, tienen como propósito reconocer la realidad de la cultura curricular de la UPEL.

Así, se tiene que solo una minoría es identificada como hacedora de currículo, pero no de teoría curricular. Ello devela el evidente predominio de una racionalidad técnica por encima de una racionalidad teórica, tan necesaria para consolidar la apropiación del saber curricular y recuperar el potencial de la dinámica curricular en la UPEL. Muestra de ello se encuentra en la concepción de currículo de 1996, de acuerdo con la cual "el currículo se constituye dentro de una estructura conformada por cuatro componentes curriculares articulados e integrados a partir de la concepción del componente como el conjunto de acciones y experiencias que se agrupan en cursos, fases y actividades de extensión" (UPEL, 1996: 35). Tal estructura fue diseñada por los expertos del momento, pero desconocida por el 90\% de los actores según el Informe de Evaluación elaborado por la Comisión de Currículo de Pregrado en el año 2005.

Cabe destacar que la evaluación curricular en la UPEL es declarada oficialmente abierta por el Consejo Universitario en el año 2002 mediante la Resolución $\mathrm{N}^{\mathrm{o}}$ 2002.238.209 de fecha 22/04/02, pero sus resultados fueron presentaron al Consejo Universitario en el año 2004 por el Vicerrectorado de Docencia. Entre los hallazgos se evidenció la pertinencia de muchos de los planteamientos de la fundamentación teórica expuesta en el Diseño Curricular, Documento Base de 1996, pero también, la falta de articulación entre lo teórico y la implementación y administración curricular del 
mismo, expuestos en los testimonios presentados como evidencia mostrada por los actores.

Las debilidades encontradas en los planos pedagógico y curricular como resultado de la evaluación curricular realizada permiten comprobar que existe en desfase comunicativo entre los expertos en currículo y el resto de la comunidad universitaria de la UPEL ("a la gente de currículo nadie le entiende"). A ello se suma la falta de comprensión de la actividad curricular en la Universidad como resultado de la deficiente comunicación entre los docentes y los encargados del currículo en la institución. En el cuadro que se presenta a continuación se puede observar que existen muchas debilidades en el Diseño Curricular de 1996, las cuales están relacionadas con la poca claridad existente. En efecto, no existe comprensión entre los profesores de lo que pretendieron expresar los curricultores. Con este diseño con debilidades tan marcadas, es evidente que la actividad curricular se ve afectada y en consecuencia, deteriorada y poco pertinente.

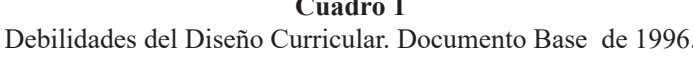

Plano Pedagógico Plano Curricular

- Incongruencia entre la concepción educativa que sustenta el Documento Base del Diseño Curricular (1999) y las prácticas pedagógicas.

- Carencia de un programa de desarrollo del personal docente de la UPEL que responda con efectividad de la acción docente.

- Falta de acompañamiento al docente para promover la apropiación crítica de la concepción curricular presente.

- Poca duración de la práctica profesional, acompañada de su escasa vinculación a las exigencias de los niveles y modalidades del sistema educativo venezolano así como su incorporación tardía.

- Escasa aplicación de la investigación en las prácticas docentes.

- Desvinculación entre la formación pedagógica y la formación especializada, con privilegio de esta última.

- Presencia de una evaluación del aprendizaje que privilegia el resultado y no el proceso de construcción del conocimiento ni el desarrollo de competencia y valores previstos.
- Diferencias significativas en el número de horas asignadas a los cursos y fases de los bloques homologado e institucional.

- Repetición de contenidos.

- Diferencias en la denominación y ubicación de los cursos o fases en las distintas áreas y niveles.

- Diferencias significativas en la aplicación de los criterios a considerar para determinar el carácter obligatorio u optativo de cursos o fases del bloque institucional.

- Existencia de prelaciones injustificadas y excesivas.

- Falta de correspondencia entre los planes de estudio y el perfil de egreso.

- Modificaciones a los planes de estudio sin autorización del Consejo Universitario.

- Inadecuada administración de las fases y de los diferentes cursos del eje didáctico.

- Desarticulación en la administración de los com- 
- Ausencia de un trabajo pedagógico interdisciplinario que permita el intercambio y la confrontación de los saberes disciplinares. ponentes de Formación General, Especializada, Pedagógica y Práctica Profesional.

- Falta de conexión entre conocimientos, habilidades, destrezas, actitudes y aptitudes requeridas para el ejercicio docente durante el proceso formativo.

- Ignorancia de los ejes transversales en el diseño curricular.

Fuente: Elaboración propia a partir de datos contenidos en el Informe de Evaluación curricular de 2005 presentado por la Comisión de Currículo de Pregrado de la UPEL.

Con la recuperación del potencial de la realidad curricular en la UPEL, se espera reconocer la pluralidad de categorías referenciales a ser consideradas en el proceso de transformación curricular conducido por el Vicerrectorado de Docencia desde 2009 y llevado a concreción por la Comisión de Currículo de Pregrado e integrada por los Jefes de las Unidades de Currículo de cada uno de los Institutos Pedagógicos.

Tales categorías referenciales son: innovación, investigación, extensión y gestión, dejando claro que aun cuando todos los actores se hicieron visibles como consumidores de teorías curriculares y no como creadores de estas, se emprende una estrategia que viabiliza la posibilidad de poner el conocimiento curricular al alcance de todos los actores. Dicha estrategia es configurada como respuesta al requerimiento institucional de poseer un nuevo currículo y en consecuencia, nuevos diseños curriculares, para lo cual se genera la nueva concepción de currículo que asume la UPEL, denominada currículo como espacio público.

\section{Construcción teórica derivada del análisis e interpretación de los resultados}

La necesidad de empoderar a los actores del discurso curricular constituyó la base de un proceso de dinamización de la actividad curricular que promoviera y generara estrategias a partir de las cuales fue posible consensar y asumir la propuesta de Fernández (2008) de concebir el currículo como espacio público, de construcción colectiva, generada desde el acontecer cotidiano y desde las experiencias de los actores. Es realmente un reconocimiento al haber generado desde el diálogo de saberes y el intercambio de experiencias, propio de una institución universitaria formadora de docentes como lo es la UPEL.

Al consensuar la UPEL el currículo como espacio público se fortalece desde el Vicerrectorado de Docencia, (instancia responsable del currículo de pregrado), la transformación de la estructura curricular de la formación docente, pues desde este ámbito se promueve la participación de todos en la planificación, construcción, diseño, desarrollo y evaluación curricular, independientemente de las características particulares de cada uno de ellos, de la forma de organización en la que se ubiquen o del grupo social, político y/o cultural al cual pertenezcan. Sin embargo enfrenta el desafío de lograr que todos comprendan la importancia de su participación en el escenario curricular y del dominio del discurso propio de este campo de conocimiento, accesible a todos.

Esto lleva a considerar que la gestión integral del currículo debe ser abierta y descentralizada en y para la toma de decisiones, además de procurar una redistribución de las responsabilidades en el diseño de innovaciones con mayor autonomía, concreción de acciones y diferenciación en los niveles de participación libre, responsable y voluntaria como 
forma práctica de hacer desarrollo curricular y de revalorizar los espacios de participación existentes en cada uno de los Institutos que conforman la Universidad, apoyados en los Jefes de las Unidades de Currículo, quienes como ya se indicó, conforman la Comisión de Currículo de Pregrado dependiente del Vicerrectorado de Docencia.

Todo lo anterior pretende dar respuesta a los enfoques y concepciones que tradicionalmente se han desarrollado en la UPEL: (a) académico: con énfasis en los valores culturales y en la formación intelectual; (b) tecnológico: con énfasis en cómo debe ser presentar la información en los medios y métodos; (c) social-re construccionista: destaca fundamentalmente las necesidades sociales por encima de las necesidades individuales; y (d) cognitivo: con valorización de los procesos cognitivos y en el aprendizaje por descubrimiento, siempre trabajados desde la interpretación de los expertos en el campo curricular.

De acuerdo con los señalamientos de los estudiantes, se profundiza en la importancia del componente especializado y se menosprecia el componente general y pedagógico que conforman el Diseño Curricular de 1996. Así se constata en los siguientes testimonios de estudiantes del Instituto Pedagógico de Caracas: "Los profesores enseñan solo contenidos disciplinares", "Las materias pedagógicas son de relleno". Opiniones semejantes se encuentran también entre los alumnos del Instituto Pedagógico Rural El Mácaro: "Lo más importante es la especialidad"; lo mismo que en el Instituto Pedagógico Rural Gervasio Rubio: "En la UPEL es un problema formar docentes que sepan de la especialidad si ocupan mucho tiempo en otras materias".

Lo anterior permite establecer que tradicionalmente se han enseñado los contenidos desde la estructura de las disciplinas y esa enseñanza constituye una forma de aproximación a los criterios de verdad que manejan los docentes, empleando en ocasiones recursos tecnológicos que pretenden facilitar el aprendizaje de dichos contenidos, pero sin la comprensión del para qué de esos contenidos en el contexto de la formación docente, carentes de valoraciones significativas aunque rigurosamente organizados.

De las afirmaciones anteriores surge un nuevo desafío para la UPEL: promover una nueva visión de la enseñanza como categoría emergente orientada hacia la consolidación del nuevo enfoque curricular centrado en el desarrollo de competencias y del modelo de formación centrado en el desarrollo humano, Documento Base (2011), acompañado de una didáctica crítica, centrada en cómo se aprende y no en cómo se enseña, es decir, con énfasis en el aprendizaje y no en la enseñanza. Para ello se requiere del análisis del imaginario plasmado en la producción de quienes marcan la pauta del saber didáctico y pedagógico en la UPEL. Se trata, finalmente, de recuperar saberes y experiencias para diseñar propuestas pedagógicas concertadas.

\section{Aproximación conclusiva}

En este momento de cambios sociales, tecnológicos y educativos, se hace necesario en el seno de la UPEL movilizar las instancias decisorias para que la concepción de currículo asumida por la institución sea comprendida y compartida por todos los actores con la finalidad de recuperación del saber pedagógico en y para la praxis pedagógica participativa y protagónica. Esta participación resulta fundamental para consolidar un cambio institucional reciente, caracterizado por la expansión del acceso a la cultura curricular, que estuvo restringida por la élite de intelectuales que impregnó la dinámica curricular de las instituciones universitarias, hasta finales del siglo XX, manteniendo una forma de exclusión amparada bajo la figura del "saber especializado".

Es así como en la UPEL se propicia una verdadera transformación curricular bajo el supuesto de que el currículo se aprende haciendo currículo, es decir, practicando, teorizando y practicando constantemente. Se llega de este modo a asumir el compromiso de sistematizar la producción teórica en el ámbito curricular como categoría emergente que 
ejerció y ejerce presión en la toma de decisiones y en las relaciones de poder vinculadas a la estructura de la universidad.

Estas consideraciones parten de la revisión de perspectivas teóricas, ideológicas, técnicas, sociales y éticas en y desde la evolución de las corrientes del pensamiento pedagógico que caracterizan a la UPEL, pero que la cuestionan en su esencia al asumir que el currículo como espacio público se dinamiza al atender expectativas, necesidades, intereses y aportes de diferentes actores en diferentes contextos, necesitando para ello de criterios que permitan definir de forma conjunta perfiles, componentes, competencias, saberes y estrategias, que sean claramente comprendidos por todos los actores y no solo por unos cuantos conocedores o expertos en el área de conocimiento.

De allí surge la necesidad de generar criterios para la comprensión didáctica y contextual del currículo como espacio público en la UPEL, con la finalidad de contribuir con la gestión integral del proceso formativo de los ciudadanos, profesionales e investigadores y ahora, incorporando la idea de promover la distribución del poder en la construcción del diseño curricular de la carrera docente y áreas afines con sentido social, político, cultural, histórico y ético. Todo lo anterior constituye un desafío para la comunidad upelista, que debe asumir la conducción del proceso curricular en dos vías: individual y colectiva. Hasta ahora la reflexión curricular en el contexto universitario ha estado reservada a unos pocos, pero esta visión debe cambiar en función de los desafíos del siglo XXI, que comprometen la construcción de una nueva ciudadanía, con visión anticipada de la realidad compleja abordada con elementos conceptuales y metodológicos que permitan su reconfiguración.

\section{Referencias}

Becerra, A. (1974). La revolución científico-técnica y la revolución en la educación. Gaceta de Pedagogía, 24. Caracas: Instituto Pedagógico de Caracas.
Becerra, A. (2004) Thesaurus curricular universitario. Caracas: Sin publicar.

Brunner, J. (2014). Tendencias de la educación superior a nivel mundial.Centro de Políticas Comparadas en Educación. Ponencia, noviembre. UNESCO.

Fernández, B. (2006). Sistematización de la investigación ante la impunidad curricular y su impacto en la modernización y transformación del currículo. Ponencia presentada en XIII Jornada Anual de Investigación y IV Jornada de Postgrado. IPC-UPEL. Caracas, 30 de octubre hasta el 3 de noviembre.

Fernández, B. (2007). Sistematización de la investigación y su impacto en el currículo de formación docente. Vía para la integración en la Universidad Pedagógica Experimental Libertador. Ponencia presentada en la Semana Aniversario del Departamento de Curriculum y Administración. Cumaná: Universidad de Oriente.

Fernández, B. (2008).Papel de la pedagogía en el currículo. Instituto de Altos Estudios Diplomáticos.

Ministerio de Educación Cultura y Deportes. (2001).Políticas y estrategias para el desarrollo de la Educación Superior en Venezuela 20002006. Caracas: Publicaciones del Ministerio de Educación, Cultura y Deportes.

República Bolivariana de Venezuela (1999). Constitución. Caracas: Publicaciones de la Asamblea Nacional.

República Bolivariana de Venezuela. (2000). Ley Orgánica de Educación(2000).Caracas: Publicaciones de la Asamblea Nacional.

Universidad Pedagógica Experimental Libertador. (1996).Diseño Curricular. Documento Base. Caracas: Fondo Editorial de la Universidad Pedagógica Experimental Libertador.

Universidad Pedagógica Experimental Libertador. (2011). Documento Base del Currículo 2011. Caracas: Fondo Editorial de la Universidad Pedagógica Experimental Libertador. 
Zemelman, H. (2006). El conocimiento como desafío posible. México D.F.: Instituto Politécnico Nacional. México.

Zemelman, H. (2011). El arte de pensar los maestros. El proceso de formación y la conciencia histórica en América. Cumaná: Universidad de Oriente. 